\title{
Dirac-Hartree-Bogoliubov Approximation for Finite Nuclei with Blocking
}

\author{
E. Baldini-Neto, B. V. Carlson, \\ Departamento de Física, Instituto Tecnológico de Aeronáutica, \\ Centro Técnico Aeroespacial, 12228-900 São José dos Campos, São Paulo, Brazil \\ and D. Hirata \\ Department of Physics and Astronomy, The Open University, \\ Milton Keynes Mk7 6AA, Buckinghamshire, United Kingdom
}

Received on 03 October, 2003

\begin{abstract}
We have extended the Dirac-Hartree-Bogoliubov (DHB) approximation developed in a previous work [1] with the inclusion of blocking terms in order to study the pairing properties of both even and odd nuclei. We have concentrated our attention in the isotope chains of ${ }^{12-26} \mathrm{O},{ }^{34-56} \mathrm{Ca},{ }^{48-78} \mathrm{Ni}$ and ${ }^{100-132} \mathrm{Sn}$ as well as on the $\alpha$-decay of the new superheavy element ${ }^{277} 112$.
\end{abstract}

\section{Introduction}

The development of new facilities with the aim of studying unstable nuclei has enabled experimental measurements of masses, radii and deformations of such systems in a wider region of the nuclear chart. Studies in this 'terra exotica' have revealed new features such as neutron halos or skins $[2,3,4]$ and brought new perspectives to nuclear physics.

Relativistic many-body theories have been applied to nuclei and nuclear matter with remarkable success $[5,6,7]$. In a previous work [1], the Dirac-Hartree-Fock-Bogoliubov (DHFB) approximation was developed to describe the ground state wave functions and energies of finite nuclei. It was applied to spin-zero proton-proton and neutron-neutron pairing within the Dirac-Hartree-Bogoliubov (DHB) approximation (the exchange term was neglected) using a zero-range approximation to the pairing tensor. The latter can be justified by demonstrating that the effective length for spatial variations of the wavefunctions, in the calculations performed, is much larger than the range of the non-locality of the pairing tensor, rendering the spatial variations of the wave function close to negligible within the range of pairing non-locality. The Dirac structure of the pairing field is retained in the DHFB approximation. The resulting pairing field is dominated by a scalar term and the zero component of a vector term, as found in the case for ${ }^{1} S_{0}$ pairing in symmetric nuclear matter.

\section{Dirac-Hartree with blocking}

The Lagrangian density can be written as:

$$
\mathcal{L}=\mathcal{L}_{0}+\mathcal{L}_{\text {int }}
$$

where $\mathcal{L}_{0}$ is the free Lagrangian density given by

$$
\begin{aligned}
\mathcal{L}_{0}= & \left.\bar{\Psi}(x)[i D-M] \Psi(x)+\frac{1}{2} \partial_{\mu} \sigma(x) \partial^{\mu} \sigma(x)\right]-U(\sigma(x))-\frac{1}{4} F_{\mu \nu} F^{\mu \nu} \\
& -\frac{1}{4} \Omega_{\mu \nu} \Omega^{\mu \nu}+\frac{1}{2} m_{\omega}^{2} \omega_{\mu}(x) \omega^{\mu}(x)-\frac{1}{4} \vec{G}_{\mu \nu} \cdot \vec{G}^{\mu \nu}+\frac{1}{2} m_{\rho}^{2} \vec{\rho}_{\mu}(x) \cdot \vec{\rho}^{\mu}(x)
\end{aligned}
$$

with

$$
\begin{aligned}
& F_{\mu \nu}=\partial_{\mu} A_{\nu}-\partial_{\nu} A_{\mu}, \\
& \Omega_{\mu \nu}=\partial_{\mu} \omega_{\nu}-\partial_{\nu} \omega_{\mu}, \\
& \vec{G}_{\mu \nu}=\partial_{\mu} \vec{\rho}_{\nu}-\partial_{\nu} \vec{\rho}_{\mu},
\end{aligned}
$$

and $U(\sigma(x))$ being a non-linear potential given in Ref.[1].

The interaction terms in the Lagrangian density are taken to have the simplest possible form consistent with their Lorentz and isospin structure, 


$$
\begin{aligned}
& \mathcal{L}_{\text {int }}=g_{\sigma} \bar{\Psi}(x) \sigma(x) \Psi(x)-g_{\omega} \bar{\Psi}(x) \gamma_{\mu} \omega^{\mu}(x) \Psi(x)-\frac{1}{2} g_{\rho} \bar{\Psi}(x) \gamma_{\mu} \vec{\tau} \cdot \vec{\rho}^{\mu}(x) \Psi(x) \\
& -e \bar{\Psi}(x) \frac{\left(1+\tau_{3}\right)}{2} \gamma_{\mu} A^{\mu}(x) \Psi(x) .
\end{aligned}
$$

The DHFB approximation is obtained by characterizing the average effect of the interaction of a nucleon with the other nucleons through an effective single particle Lagrangian, $L_{e f f}$, given in terms of the self-energy, which describes the average interaction of a nucleon with the sur- round matter and the pairing field $\Delta$ (and its conjugate $\bar{\Delta}$ ) which describes the creation (annihilation) of a pair during the propagation. It was shown in Ref.[1] that these fields satisfy the following self-consistency equations,

$$
\begin{aligned}
& \Sigma(\vec{x}, \vec{y})=\delta(\vec{x}-\vec{y}) \sum_{j} \Gamma_{j \alpha} \int d^{3} z D_{j}^{\alpha \beta}(\vec{x}-\vec{z}) \sum_{\epsilon_{\gamma}<0} \bar{U}_{\gamma}(\vec{z}) \Gamma_{j \beta}(\vec{z}) U_{\gamma}(\vec{z}) \\
& -\sum_{j} \Gamma_{\alpha}(\vec{x}) D^{\alpha \beta}(\vec{x}-\vec{y}) \sum_{\epsilon_{\gamma}<0} U_{\gamma}(\vec{x}) \bar{U}_{\gamma}(\vec{y}) \Gamma_{j \beta},
\end{aligned}
$$

and

$$
\Delta(\vec{x}, \vec{y})=-\sum_{j} \Gamma_{j \alpha}(\vec{x}) D_{j}^{\alpha \beta}(\vec{x}-\vec{y}) \sum_{\epsilon_{\gamma}<0} U_{\gamma}\left(\vec{x} \bar{V}_{\gamma}(\vec{y}) A \Gamma_{j \beta}^{T}(\vec{y}) A^{\dagger},\right.
$$

where the sum runs over the negative frequency solutions, $\epsilon_{\gamma}<0$.

The generalized baryon (quasi-particle) propagator is

$$
\mathbf{S}(\tilde{\mathbf{x}}, \tilde{\mathbf{y}} ; \omega)=\left(\begin{array}{ll}
G(\vec{x}, \vec{y} ; \omega) & F(\vec{x}, \vec{y} ; \omega) \\
\tilde{F}(\vec{x}, \vec{y} ; \omega) & \tilde{G}(\vec{x}, \vec{y} ; \omega)
\end{array}\right)
$$

and can be written as

$$
\mathbf{S}(\tilde{\mathbf{x}}, \tilde{\mathbf{y}} ; \omega)=\sum_{\alpha}\left(\begin{array}{c}
U_{\alpha}(\vec{x}) \\
V_{\alpha}(\vec{x})
\end{array}\right) \frac{1}{\omega-\epsilon_{\alpha}+\mathbf{i} \eta}\left(\bar{U}_{\alpha}(\vec{y}) \quad \bar{V}_{\alpha}(\vec{y})\right)+\sum_{\beta}\left(\begin{array}{c}
U_{\beta}(\vec{x}) \\
V_{\beta}(\vec{x})
\end{array}\right) \frac{1}{\omega+\epsilon_{\beta}-\mathbf{i} \eta}\left(\bar{U}_{\beta}(\vec{y}) \quad \bar{V}_{\beta}(\vec{y})\right)
$$

with the components $U_{\alpha, \beta}$ and $V_{\alpha, \beta}$ being Dirac spinors corresponding to the normal and time-reversed components of the positive (negative) frequencies, $\epsilon_{\alpha}\left(\epsilon_{\beta}\right)$ respectively, of the Dirac-Gorkov equation whose solutions occur in pairs with real eigenvalues of opposite signs. The pairs of eigenvectors, for neutrons or protons, are related as.

$\epsilon=\epsilon_{\alpha}:\left(\begin{array}{l}U(\vec{y}) \\ V(\vec{y})\end{array}\right)$

$$
\epsilon=-\epsilon_{\alpha}:\left(\begin{array}{c}
-\gamma_{0} B V^{*}(\vec{y}) \\
\gamma_{0} B U^{*}(\vec{y})
\end{array}\right) .
$$

where $B=\gamma^{5} C$.

In order to treat odd systems we have performed Pauliblocking of selected single-particle states, interchanging one of a pair of eigenvectors by its time-reversed state. This corresponds to the following transformation

$$
\left(\begin{array}{c}
U(\vec{y}) \\
V(\vec{y})
\end{array}\right) \rightarrow\left(\begin{array}{c}
-\gamma_{0} B V^{*}(\vec{y}) \\
\gamma_{0} B U^{*}(\vec{y})
\end{array}\right)
$$

which modifies the densities that enter in the Hartree terms of the self-energy given in equation (5) and annuls the contribution of the blocked states to the pairing field, given in equation (6).

\section{Results}

As a first test of our approach, we have calculated the mass deffects of the ${ }^{12-26} \mathrm{O},{ }^{34-56} \mathrm{Ca},{ }^{48-78} \mathrm{Ni}$ and ${ }^{100-132} \mathrm{Sn}$ isotope chains using the Dirac-Hartree-Bogoliubov approximation (DHB) with blocking, treating the pairing correlations through the Dirac-BCS approximation. The results are labled by (DHB+BCS) in figures (3)-(3) and are compared to the experimental results of Ref. [8]. 
O Isotopes

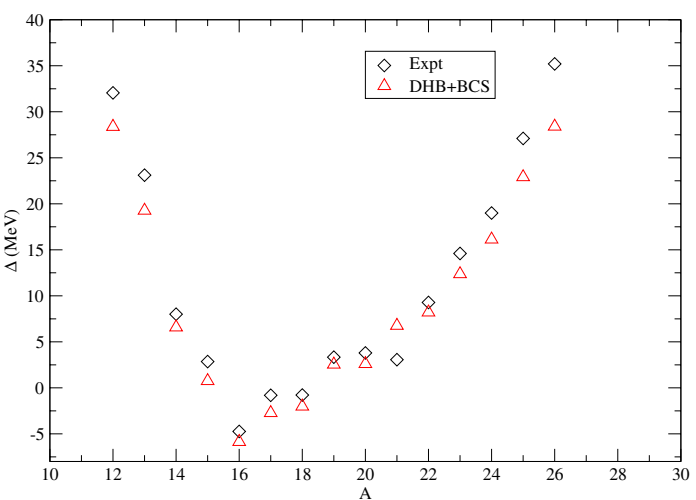

Figure 1. DHB+BCS calculations for mass deffects of the ${ }^{12-26} \mathrm{O}$ $(\mathrm{Z}=8)$ isotope chain compared with the experimental values of Ref. $[8]$.

Ca Isotopes

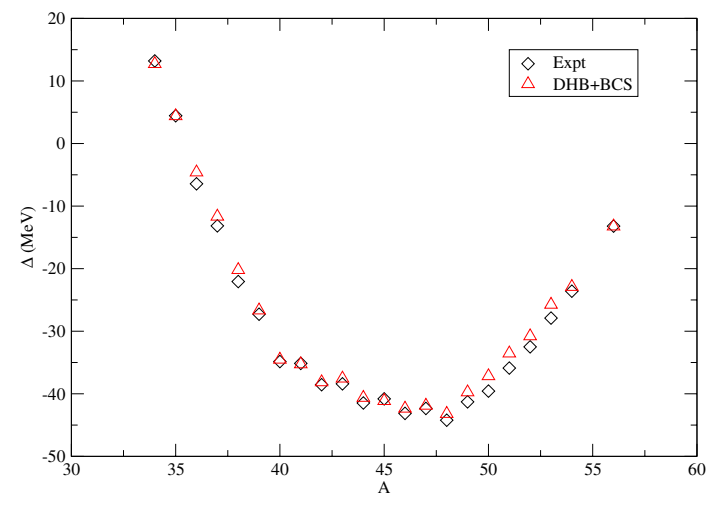

Figure 2. DHB+BCS calculations for mass deffects of the ${ }^{34-56} \mathrm{Ca}$ $(\mathrm{Z}=20)$ isotope chain compared with the experimental values of Ref. [8].

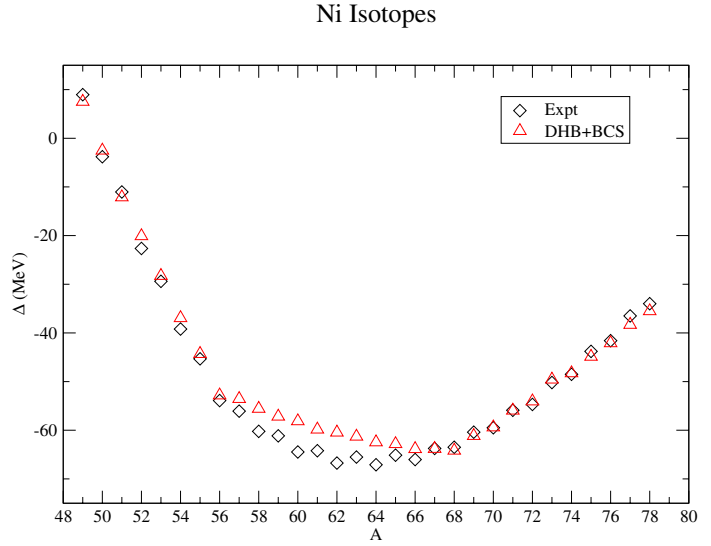

FIgure 3. DHB+BCS calculations for mass deffects of the ${ }^{48-78} \mathrm{Ni}$ $(\mathrm{Z}=28)$ isotope chain compared with the experimental values of Ref. [8].

Sn Isotopes

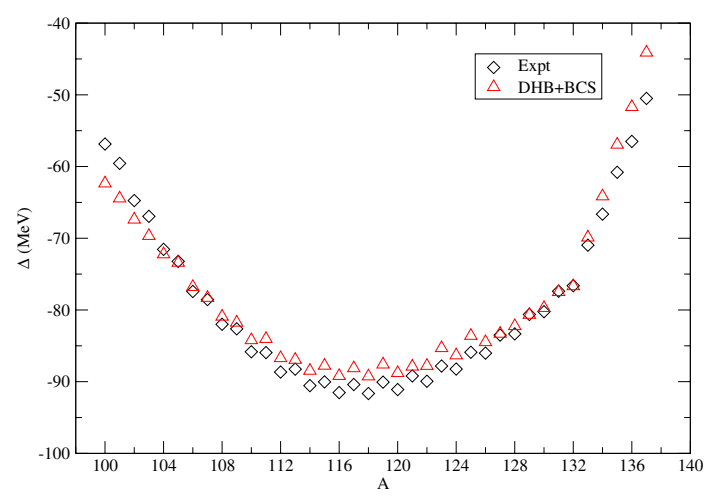

Figure 4. DHB+BCS calculations for mass deffects of the ${ }^{100-132} \mathrm{Sn}(\mathrm{Z}=50)$ isotope chain compared with the experimental values of Ref. [8].

We have also calculated the $\alpha$-decay chain of of the new superheavy element ${ }^{277} 112$ [9] within the blocked+DHB formalism. The results, compared to those of a nonrelativistic Hartree-Fock-Bogoliubov approach, which considers a Gogny force (HFB+Gogny), and also to the experimental data of Ref. [8], are shown in Table 1. As can be seen, the results are very promising.

TABLE 1. $\alpha$-decay chain of ${ }^{277} 112$ calculated in blocked+DHB, HFB+Gogny compared with the experimental data of Ref. [8].

\begin{tabular}{crrr}
\hline \hline mother $\rightarrow$ daughter & gs $\rightarrow$ gs & gs $\rightarrow$ gs & expt $(\mathrm{MeV})$ \\
\hline \hline & blocked+DHB & HFB+Gogny & \\
\hline \hline${ }^{277} 112 \rightarrow{ }^{273} 112$ & 12.246 & 12.323 & $11.45-11.65$ \\
\hline${ }^{273} 110 \rightarrow{ }^{269} \mathrm{Hs}$ & 10.187 & 11.266 & $9.73-11.08(?)$ \\
\hline${ }^{269} \mathrm{Hs} \rightarrow{ }^{265} \mathrm{Sg}$ & 10.099 & 9.034 & $9.17-9.23(?)$ \\
\hline${ }^{265} \mathrm{Sg} \rightarrow{ }^{261} \mathrm{Rf}$ & 8.462 & 9.084 & 8.77 \\
\hline${ }^{261} \mathrm{Rf} \rightarrow{ }^{257} \mathrm{No}$ & 6.711 & 8.487 & 8.52 \\
\hline${ }^{257} \mathrm{No} \rightarrow{ }^{253} \mathrm{Fm}$ & 7.068 & 8.048 & $8.34-8.45$ \\
\hline${ }^{253} \mathrm{Fm} \rightarrow{ }^{249} \mathrm{Cf}$ & 8.308 & 7.446 & 7.197 \\
\hline${ }^{249} \mathrm{Cf} \rightarrow{ }^{245} \mathrm{Cm}$ & 6.350 & 6.350 & 6.295 \\
\hline \hline
\end{tabular}

\section{Conclusions and Perspectives}

We have included Pauli-blocking terms in the DiracHartree-Bogoliubov approach previously developed in Ref.
[1]. As a initial test we have calculated the mass deffects of the isotope chains of $\mathrm{O}, \mathrm{Ca}, \mathrm{Ni}$ and $\mathrm{Sn}$ and the $\alpha$-decay chain of the superheavy element ${ }^{277} 112$. We have obtained very reasonable results compared to the experimental data 
in the former case, and with a non-relativistic calculation as well in the latter case. As a next step, after repeating the calculations shown in this work within the full DHFB approximation, we intend to apply the formalism along the nuclear chart, giving special attention to light neutron-rich isotopes such as those of $\mathrm{Li}, \mathrm{Be}, \mathrm{B}, \mathrm{C}$ and $\mathrm{N}$.

EBN acknowledges the support of Fundação de Amparo à Pesquisa do Estado de São Paulo (FAPESP). BVC acknowledges partial support from FAPESP and from the Conselho Nacional de Pesquisa e Desenvolvimento (CNPq).

\section{References}

[1] B. V. Carlson and D. Hirata, Phys. Rev. C 62, 054310 (2000).
[2] I. Tanihata et al. Phys. Rev. Lett. 55, 2676 (1985).

[3] I. Tanihata, D. Hirata, T. Kobayashi, S. Shimoura, K. Sugimotoand H. Toki, Phys. Lett. B 289, 261 (1992).

[4] T. Suzuki et al. Phys. Rev. Lett. 75, 3241 (1995).

[5] B. D. Serot and J. D. Walecka, Adv. Nucl. Phys. 16, 1 (1986).

[6] P. G. Reihardt, Rep. Prog. Phys. 52, 439 (1989).

[7] B. D. Serot, Rep. Prog. Phys. 55, 1855 (1992).

[8] G. Audi, O. Bersillon, J. Blachot and A. H. Wapstra, Nucl. Phys. A 729, 3-128 (2003).

[9] P. J. Karol et al., Pure Appl. Chem. 75, 1601 (2003). 